Cite this: Phys. Chem. Chem. Phys., 2013, 15, 16065

Received 29th July 2013, Accepted 5th August 2013 DOI: $10.1039 / \mathrm{c} 3 \operatorname{cp} 53115 \mathrm{e}$

www.rsc.org/pccp

\title{
From hydrogen bond donor to acceptor: the effect of ethanol fluorination on the first solvating water molecule $\dagger$
}

\author{
Matthias Heger, Tina Scharge $\ddagger$ and Martin A. Suhm*
}

\begin{abstract}
Supersonic jet FTIR spectra of the $\mathrm{OH}$ stretching vibrations in complexes of mono-, di- and trifluoroethanol with water are presented. In contrast to the non-fluorinated ethanol case, the fluorinated alcohols are all shown to act as $\mathrm{O}-\mathrm{H}$... O hydrogen bond donors towards water. This is found to be mostly a consequence of the intramolecular electron-withdrawing effect of the fluorine atoms and, with decreasing importance for increasing fluorination, due to the attractive intermolecular contact between one of the dangling water $\mathrm{OH}$ groups and the fluorine atoms. The findings provide a stepwise rationalization for the hydrophobic properties of the pharmaceutically important trifluoromethyl group.
\end{abstract}

\section{Introduction}

Supramolecular design rests on differentiation and control of intermolecular interactions such as hydrogen bonds. ${ }^{1}$ By studying the simplest model systems of their kind, useful insights for more complex structures can be obtained. ${ }^{2-6}$ Such a simple model system is the mixed dimer of ethanol and water, ${ }^{7}$ in which the water molecule is known to act preferentially as the hydrogen bond donor. The explanation is straightforward in terms of inductive effects: an ethyl group increases the electron density at the alcohol oxygen, more so than a hydrogen atom. Therefore, ethanol is a better hydrogen bond acceptor than water. It is actually also predicted to be a better hydrogen bond donor than water, ${ }^{8}$ but the former superiority prevails. ${ }^{7}$ The experimental proof of this donor-acceptor preference is quite recent: a Raman spectroscopic study ${ }^{7,9}$ has shown that in the cold ethanol-water dimer, water acts as a donor and ethanol switches from its preferred trans structure to a gauche conformation. This preference in conformation and coordination is also predicted by many quantum-chemical calculations, ${ }^{7,10-13}$ which show that the energy penalty for the inverted complex with water as an acceptor is not very large, on the order of $3-4 \mathrm{~kJ} \mathrm{~mol}^{-1}$.

How can this energy sequence be turned around? Again, the answer is straightforward: the electron density at the alcohol oxygen has to be decreased. At the same time, new binding

Institute of Physical Chemistry, Georg-August-Universität, Tammannstraße 6, 37077 Göttingen, Germany. E-mail: msuhm@gwdg.de

$†$ Electronic supplementary information (ESI) available: Complete basis set extrapolations, 1-dimensional vibrational analysis and water vibrations. See DOI: $10.1039 / \mathrm{c} 3 \mathrm{cp} 53115 \mathrm{e}$

‡ Current address: Gesellschaft für Anlagen- und Reaktorsicherheit (GRS) mbH, Schwertnergasse 1, 50667 Köln, Germany. options may be offered to a dangling $\mathrm{OH}$ bond of the acceptor water. Both requirements can be fulfilled simultaneously by replacing some of the terminal $\mathrm{C}-\mathrm{H}$ bonds in ethanol by $\mathrm{C}-\mathrm{F}$ bonds. The electronegative fluorine reduces the electron donating power of the ethyl group and offers a secondary hydrogen bond acceptor site for one of the coordinating water $\mathrm{O}-\mathrm{H}$ groups. This $\mathrm{O}-\mathrm{H} \cdots \mathrm{O}-\mathrm{H} \cdots \mathrm{F}$ motif is cooperative, in contrast to the alternative $\mathrm{O} \cdots \mathrm{H}-\mathrm{O}-\mathrm{H} \cdots \mathrm{F}$ arrangement, in which the water molecule acts as a double donor. A similar secondary bonding option is present in water complexes of 2-phenylethanol, ${ }^{14}$ where a phenyl group takes the place of the fluorine. In contrast to the latter, the phenyl group does not have a significant electron withdrawing effect on the alcoholic $\mathrm{OH}$. This is consistent with equal $\left(3678 \mathrm{~cm}^{-1}\right) \mathrm{OH}$ stretching fundamentals for trans-ethanol ${ }^{15}$ and trans-phenylethanol. ${ }^{14}$ Nevertheless, the most stable hydrate is that in which water acts as an acceptor towards the aromatic alcohol. This may be due to the fairly strong $\mathrm{O}-\mathrm{H} \cdots \pi$ hydrogen bond, which it can undergo at the same time. However, an isomer in which water acts as a donor was also observed. ${ }^{14}$ This structure is stabilized by a secondary $\mathrm{C}-\mathrm{H}$ - *water interaction from the aromatic ring; therefore, the case is less clearcut than the one expected for fluorination or for the combined effect of fluorination and phenyl substitution. ${ }^{16}$

In the present work, we investigate for the first time isolated mixed dimers between water and ethanol species with one to three terminal fluorine atoms. Comparison to the parent non-fluorinated ethanol-water dimer reveals the predicted switch between donor and acceptor roles. The origins and consequences of this switch are analyzed in detail and may ultimately help to understand anomalies in bulk mixtures of water with fluorinated alcohols. ${ }^{17}$ 


\section{Experimental techniques}

For the characterization of pure and mixed alcohol-water clusters we used the filet-jet setup. ${ }^{18,19}$ A prepared gas mixture, including helium (Air Liquide, 99.996\%), a fluorinated alcohol (2-fluoroethanol = " $\mathrm{e}^{\mathrm{M}}$,", Alfa Aesar, 95\%; 2,2-difluoroethanol = "e $\mathrm{e}^{\mathrm{D},}$, Alfa Aesar, 97\%; 2,2,2-trifluoroethanol = "e $\mathrm{e}^{\mathrm{T}}$,, ABCR, 99\%) and/or water ("w", Fluka), was expanded in $135 \mathrm{~ms}$ pulses from a $67 \mathrm{~L}$ reservoir with $0.4-1.0$ bar stagnation pressure $p_{\mathrm{s}}$ through a $600 \times 0.2 \mathrm{~mm}$ slit nozzle into a jet chamber. To keep the background pressure below 1.4 mbar during the pulses, a buffer volume of $23 \mathrm{~m}^{3}$ and a pumping system with a capacity of $2500 \mathrm{~m}^{3} \mathrm{~h}^{-1}$ were used. A hibernation time of $\approx 30 \mathrm{~s}$ between pulses was necessary to allow the background pressure to drop below 0.1 mbar. The FTIR spectra were recorded $10 \mathrm{~mm}$ downstream of the expansion at $2 \mathrm{~cm}^{-1}$ resolution with a Bruker Equinox 55 spectrometer, using an InSb detector, $\mathrm{CaF}_{2}$ beam splitter and lenses, and a 2.5-3.5 $\mu \mathrm{m}$ optical filter. All spectra shown in this study were averaged over 50 to 300 pulses. Data for ethanol $\left(\mathrm{e}^{0}\right)$ were taken from ref. 7 .

\section{Computational details}

Quantum-chemical calculations were conducted using the Orca 2.9.1 $1^{20}$ and Gaussian09 ${ }^{21}$ software packages. Structure optimizations and harmonic frequency calculations for the various fluoroethanol-water clusters were done with B3LYP (optionally including Grimme's ${ }^{22-24}$ dispersion correction, B3LYP-D3) and ab initio MP2 methods, using the $6-311++\mathrm{G}(\mathrm{d}, \mathrm{p})$ Pople basis set. They yield reasonable estimates for the dimer structures and their relative stabilities and they support the interpretation of the recorded IR spectra. Furthermore, these methods can be scaled up to larger fluoroalcohols, which are among our future target molecules. We focus on mixed dimers based on the most stable fluoroethanol monomers in which the $\mathrm{OH}$ group is in a gauche conformation relative to the alkyl chain.

Because counterpoise correction of the basis set superposition error (BSSE) for our small basis set MP2 approach tends to remove the error compensation with other basis set incompleteness effects (see Section 4.2), we have applied a complete basis set (CBS) extrapolation scheme including higher-order electron correlation $^{25}$ to benchmark one exemplary energy difference. For details, see the ESI. $\dagger$

\section{Results and discussion}

Earlier investigations on ethanol-water clusters ${ }^{7,9}$ have employed a nomenclature of the form "xy" for an $x$ hydrogen bond donor and $y$ acceptor, with $\mathrm{x}$ and $\mathrm{y}=$ " $\mathrm{e}$ " for ethanol and " $\mathrm{w}$ " for water. We extend this nomenclature in the present work by adding a superscript $\mathrm{z}$ to indicate the degree of fluorination in $\mathrm{e}^{\mathrm{z}}$, referring

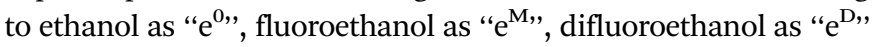
and trifluoroethanol as " $\mathrm{e}$,". Oligomers beyond the dimer are not taken into account in this work.

\subsection{Experimental spectra}

The spectroscopic probe we use for molecular recognition is the monomer $\mathrm{OH}$ stretching fundamental (denoted " $\mathrm{e}$," in Fig. 1-4), which becomes significantly red-shifted upon hydrogen bond formation. Earlier investigations by Scharge et al. ${ }^{26-29}$ provide the spectral signatures and assignments of the homodimers for all fluoroalcohols; following the general trend, the red shifts induced in the donor molecules are larger than those in the acceptors, due to a decreased bond strength of the respective donor $\mathrm{O}-\mathrm{H}$ bonds. Comparison with non-fluorinated ethanol is possible through studies by Nedic et al., ${ }^{7,9}$ which have shown spectroscopically that ethanol acts exclusively as a hydrogen bond acceptor towards water under jet-cooled conditions. Fig. 4 gives a comparison of all $\mathrm{w} / \mathrm{e}^{\mathrm{z}}$ spectra, visualizing the increasing red shift of the $\mathrm{e}^{\mathrm{z}} \mathrm{w}$ bands and the decreasing red shift of the $\mathrm{we}^{\mathrm{z}}$ band from $\mathrm{e}^{\mathrm{0}}$ to $\mathrm{e}^{\mathrm{M}}$.

Comparison of the fluoroethanol-water spectra (Fig. 1-3) with those of the pure compounds enables the identification of heterodimer bands. In all cases, a single prominent band can be identified in the mixed spectra, making the assignment straightforward. This spectral sparsity contrasts with the rich structure assigned for the homodimers, ${ }^{26-28}$ indicating more pronounced energy gaps for the heterodimers along with the lack of conformational isomerism in water. We note already here that the mixed dimer band is progressively shifted from the corresponding alcohol monomer band with increasing fluorination, indicative of increasing interaction strength.

Upon formation of the mixed dimers, the symmetric and asymmetric $\mathrm{OH}$ stretching vibrations of free monomeric water

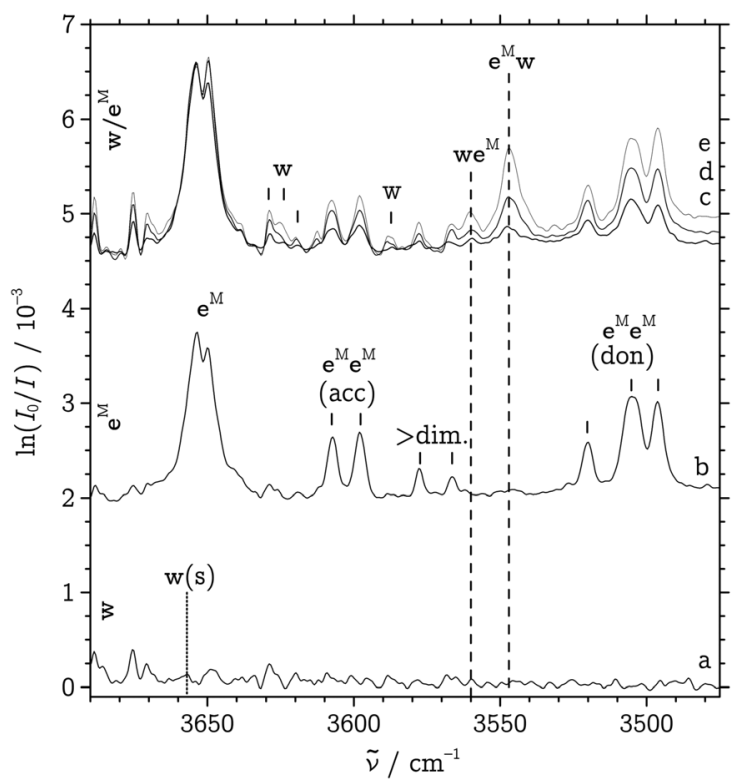

Fig. 1 Jet-FTIR spectra of $\mathrm{w}$ (trace $a$ ), $\mathrm{e}^{\mathrm{M}}(\mathrm{b})$, and $\mathrm{w} / \mathrm{e}^{\mathrm{M}}$ mixtures $(c-e)$ in helium. (a) $p_{\mathrm{s}}=1.0$ bar, $c_{\mathrm{w}}<0.05 \%$, multiplied by 5.00 , (b) $p_{\mathrm{s}}=1.0$ bar, $c_{\mathrm{e}} \mathrm{M}=0.16 \%$, (c) $p_{\mathrm{s}}=0.4$ bar, $c_{\mathrm{e}} \mathrm{m}=0.26 \%, c_{\mathrm{w}}<0.1 \%$, multiplied by 1.16 , (d) $p_{\mathrm{s}}=0.6 \mathrm{bar}$, $c_{\mathrm{e}} \mathrm{M}=0.25 \%, c_{\mathrm{w}}<0.1 \%$, and (e) $p_{\mathrm{s}}=1.0 \mathrm{bar}, \mathrm{c}_{\mathrm{e}} \mathrm{M}=0.16 \%, c_{\mathrm{w}}<0.1 \%$. "don" and "acc" indicate homodimer donor and acceptor bands, respectively; "> dim" are bands from larger homooligomers. ${ }^{26,27 ~ " ~} w^{\prime \prime}$ are rovibrational water monomer transitions in the dimer range. " $w(s)$ " marks the band center of the symmetric $\mathrm{OH}$ stretching band of monomeric water. 


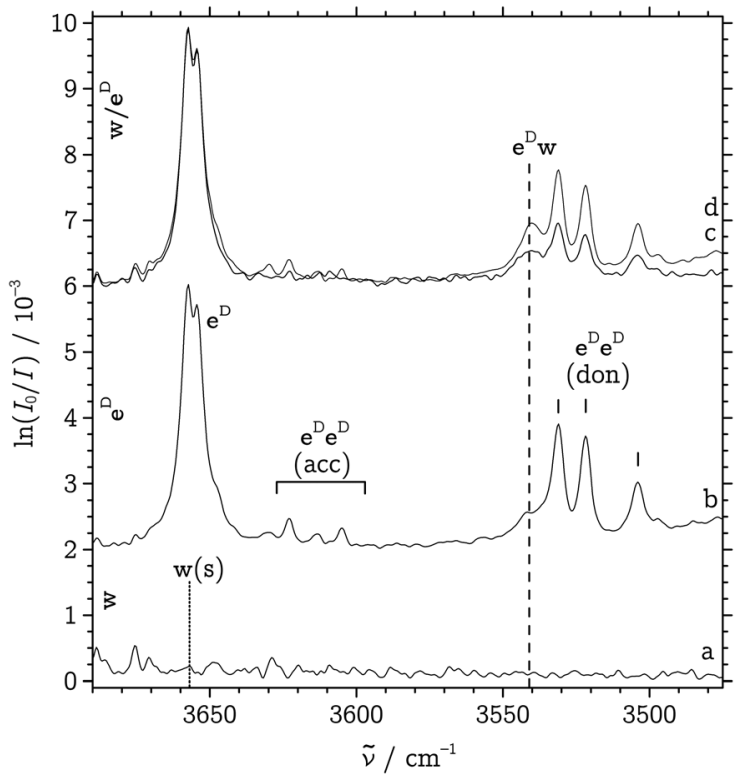

Fig. 2 Jet-FTIR spectra of $w($ trace $a), e^{D}(b)$, and $w / e^{D}$ mixtures $(c+d)$ in helium. (a) $p_{\mathrm{s}}=1.0$ bar, $c_{\mathrm{w}}<0.05 \%$, multiplied by 6.00 , (b) $p_{\mathrm{s}}=0.6$ bar, $c_{\mathrm{e}} \mathrm{D}=0.25 \%$, (c) $p_{\mathrm{s}}=0.4$ bar, $c_{\mathrm{e}} \mathrm{D}=0.25 \%, c_{\mathrm{w}}<0.1 \%$, multiplied by 2.69 , and (d) $p_{\mathrm{s}}=0.6$ bar, $c_{\mathrm{e}} \mathrm{D}=0.25 \%, c_{\mathrm{w}}<0.1 \%$. "don" and "acc" indicate homodimer donor and acceptor bands, respectively. ${ }^{27}$ " $w(s)$ " marks the band center of the symmetric $\mathrm{OH}$ stretching band of monomeric water.

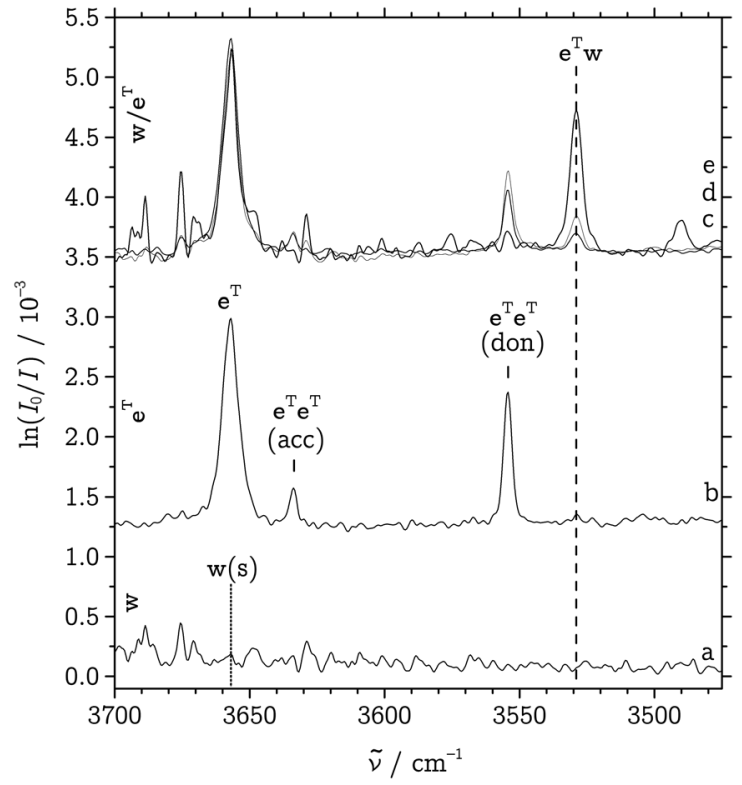

Fig. 3 Jet-FTIR spectra of $w$ (trace $a), e^{\top}(b)$, and $w / e^{\top}$ mixtures (c-e) in helium. (a) $p_{\mathrm{s}}=1.0$ bar, $c_{\mathrm{w}}<0.05 \%$, multiplied by 5.00 , (b) $p_{\mathrm{s}}=1.0$ bar, $c_{\mathrm{e}}^{\mathrm{T}}=0.02 \%$, (c) $p_{\mathrm{s}}=0.6$ bar, $c_{\mathrm{e}} \mathrm{T}=0.04 \%, c_{\mathrm{w}}<0.05 \%$, multiplied by 1.65 , (d) $p_{\mathrm{s}}=1.0$ bar, $c_{\mathrm{e}} \mathrm{T}=0.04 \%, c_{\mathrm{w}}<0.05 \%$, and (e) $p_{\mathrm{s}}=0.6$ bar, $c_{\mathrm{e}^{\mathrm{T}}}=0.04 \%, c_{\mathrm{w}}<0.1 \%$, multiplied by 2.08. "don" and "acc" indicate homodimer donor and acceptor bands, respectively. ${ }^{28}$ " $w(s)$ " marks the band center of the symmetric $\mathrm{OH}$ stretching band of monomeric water.

lose their symmetry. Still, the vibrational motions of the two protons occur in phase and out of phase of each other, respectively, and we retain the "symmetric" and "asymmetric"

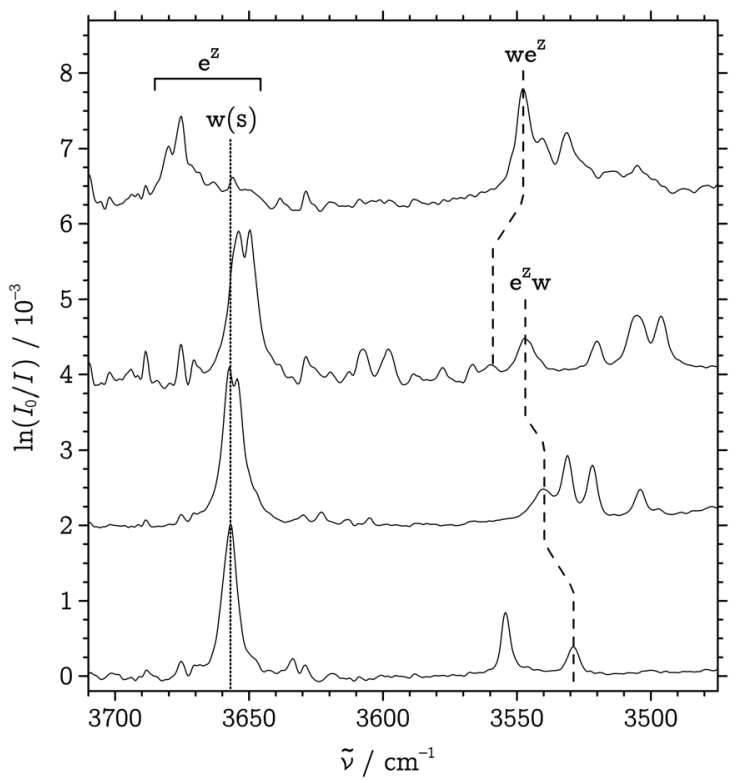

Fig. 4 Correlation of jet-FTIR spectra of $w / e^{z}$ mixtures. From top to bottom: $w / e^{0}$ (from ref. 7); w/ $/ \mathrm{e}^{\mathrm{M}}$ (trace (d) in Fig. 1); $\mathrm{w} / \mathrm{e}^{\mathrm{D}}$ (trace (d) in Fig. 2), multiplied by $0.55 ; \mathrm{w} / \mathrm{e}^{\top}$ (trace (d) in Fig. 3), multiplied by 1.20 . " $w(\mathrm{~s})$ " marks the band center of the symmetric $\mathrm{OH}$ stretching band of monomeric water.

naming with respect to these phases even for the bound water fragments, adding the descriptors in parentheses to the label where appropriate. Although the identification of the corresponding bands would be informative and increase the certainty of our assignments, several problems pertaining to their red shifts and infrared intensities arise, which we outline in the ESI. $†$ In short, we are unable to observe these bands in the mixed $\mathrm{e}^{\mathrm{D}} \mathrm{w}$ and $\mathrm{e}^{\mathrm{T}} \mathrm{w}$ spectra. For $\mathrm{e}^{\mathrm{M}} \mathrm{w}$, the symmetric stretching vibration may contribute to the triplet feature at around $3625 \mathrm{~cm}^{-1}$ attributed to water monomer transitions in Fig. 1, while we identify a possible band attributable to the asymmetric vibration in the relevant spectral region (see the ESI $\dagger$ ). These assignments are, however, not as clear-cut as for the alcoholic $\mathrm{OH}$ stretching bands.

\subsection{Comparison with calculations}

Table 1 compares the experimentally observed monomer stretching wavenumbers $\tilde{\nu}_{M}$ to the harmonic MP2/6-311++G(d,p) predictions. The experiments show all four alcohol fundamentals and the symmetric $\mathrm{w}$ stretching mode to be in close vicinity to each other, spanning a range of only $8 \mathrm{~cm}^{-1}$. The harmonic calculations succeed in reproducing both the range and sequence of these vibrations; however, the differences between theory and experiment $\left(224-228 \mathrm{~cm}^{-1}\right)$ are too large to be explained by anharmonicity effects alone. The combined anharmonicity and electronic structure gap is usually closed by wavenumber scaling.

By comparing hydrogen bond-induced red shifts observed in experiment to the corresponding harmonic predictions for dimers, one can circumvent scaling. However, the electronic structure and anharmonicity changes involved in the hydrogen bond formation process will also lead to systematic discrepancies of this quantity, even within a class of related compounds. We therefore start with a 
Table 1 Experimental anharmonic monomer $\mathrm{OH}$ stretching wavenumbers $\tilde{\nu}_{\mathrm{M}}$ and MP2/6-311++G(d,p) harmonic wavenumbers $\omega_{M}$ for water and gauche (fluoro-)ethanol monomers. For fluoroalcohols, experimental $\mathrm{OH}$ fundamentals $\tilde{\nu}_{\mathrm{e} z \mathrm{w}}$ and red shifts $-\Delta \tilde{\nu}_{\mathrm{e} z \mathrm{w}}$ in hydrogen bonds with water as an acceptor (" $\mathrm{e}^{z} \mathrm{w}^{\prime \prime}$ dimers) are also given. All data in $\mathrm{cm}^{-1}$

\begin{tabular}{lllll}
\hline & $\tilde{\nu}_{\mathrm{M}}$ & $\omega_{\mathrm{M}}$ & $\tilde{\nu}_{\mathrm{e}^{\mathrm{z}} \mathrm{w}}$ & $-\Delta \tilde{\nu}_{\mathrm{e}^{\mathrm{z}_{\mathrm{W}}}}$ \\
\hline $\mathrm{w}(\mathrm{s})$ & $3657^{30}$ & 3885 & & \\
$\mathrm{e}^{\mathrm{O}}$ & $3660^{15}$ & 3888 & & \\
$\mathrm{e}^{\mathrm{M}}$ & 3652 & 3880 & 3547 & 105 \\
$\mathrm{e}^{\mathrm{D}}$ & 3656 & 3881 & 3541 & 115 \\
$\mathrm{e}^{\mathrm{T}}$ & 3657 & 3881 & 3529 & 128 \\
\hline
\end{tabular}

systematic analysis of the heterodimer structures, energies and normal-mode wavenumbers.

For all three fluoroethanol species, the calculations predict the two most stable water heterodimer structures to be consistent: in the most stable one (Fig. 5, top), the alcohol deviates only slightly from its preferred gauche conformation and serves as a primary hydrogen bond donor towards water. The latter establishes a second, more remote contact with a fluorine atom of the alcohol, while its other hydrogen atom remains "dangling" outside the coordination ring. The second-most stable structure (Fig. 5, bottom) shows the attachment of the water molecule to a lone pair of the alcohol oxygen. The preferred site for this attachment is in the gauche position relative to the alkyl chain, ${ }^{7}$ aided in $\mathrm{e}^{\mathrm{M}}$ and $\mathrm{e}^{\mathrm{D}}$ by the formation of a secondary contact with a polarized hydrogen atom of the terminal fluoromethyl group. This compensates somewhat for the decreasing hydrogen bond acceptor strength of ethanol with increasing fluorination, leading to a weak dependence of the total binding energy on the introduction of the two first fluorines ( $c f$. also Table 2). In $\mathrm{e}^{\mathrm{T}}$, no such contact is obviously possible; the water molecule consequently "shies away" from the trifluoromethyl group, with its final position somewhat dependent on the method at use. As a consequence, the relative energy of this particular conformer is predicted to be significantly higher than that for $\mathrm{e}^{\mathrm{M}}$ or $\mathrm{e}^{\mathrm{D}}$, indicating that the secondary $\mathrm{CH} \cdots \mathrm{O}$ contact with the fluoromethyl group does indeed stabilize the structures somewhat. Following the nomenclature outlined above, the most stable (alcohol-donor) structures are of $\mathrm{e}^{\mathrm{z}} \mathrm{w}$ type, while the second-most stable structures are of $\mathrm{we}^{\mathrm{z}}$ type. Less stable water donor structures were found for $\mathrm{e}^{\mathrm{M}}$ but will not be discussed further. Overall, the energy gaps between

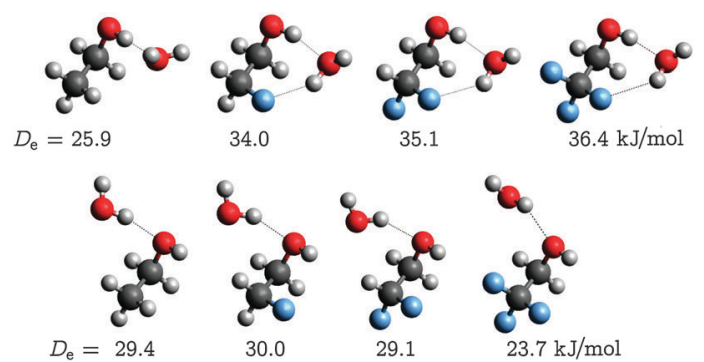

Fig. 5 Structures of $e^{z} w$ (top row) and $w e^{z}$ (bottom row) dimers for $e^{0}, e^{M}, e^{D}$ and $\mathrm{e}^{\top}$ (left to right) and electronic dissociation energies $D_{\mathrm{e}}\left(\mathrm{in} \mathrm{kJ} \mathrm{mol}^{-1}\right.$ ) at the MP2/6-311++G(d,p) level.
Table 2 Harmonically zero point-corrected dissociation energies $\left(D_{0}\right)$ and we $\mathrm{e}^{\mathrm{z}}-\mathrm{e}^{\mathrm{z}} \mathrm{w}$ energy differences $\left(\Delta E_{0}\right)$ in $\mathrm{kJ} \mathrm{mol}^{-1}$ for (fluoro-)alcohol-water dimers using the $6-311++G(d, p)$ basis set at various quantum-chemical levels. Also given are calculated red shifts $(-\Delta \omega)$ in $\mathrm{cm}^{-1}$ between free (monomer) and bonded (dimer) $\mathrm{OH}$ stretching vibrations in comparison with experiment $(-\Delta \tilde{\nu}): \mathrm{OH}$ stretch of alcohol for $e^{z} w$ and of water (in italics) for we $e^{z}$. The MP2 results for $\mathrm{e}^{\top}$ agree well with literature data ${ }^{5}$

\begin{tabular}{|c|c|c|c|c|c|c|c|c|c|c|}
\hline \multirow[b]{2}{*}{ Dimer } & \multicolumn{3}{|c|}{ B3LYP } & \multicolumn{3}{|c|}{ B3LYP-D3 } & \multicolumn{3}{|l|}{ MP2 } & \multirow{2}{*}{$\begin{array}{l}\text { Exp. } \\
-\Delta \tilde{\nu}\end{array}$} \\
\hline & $D_{0}$ & $\Delta E_{0}$ & $-\Delta \omega$ & $D_{0}$ & $\Delta E_{0}$ & $-\Delta \omega$ & $D_{0}$ & $\Delta E_{0}$ & $-\Delta \omega$ & \\
\hline \multicolumn{11}{|c|}{$\mathrm{e}^{0}$ (ethanol) } \\
\hline$e^{0} w$ & 14.8 & 0.0 & 112 & 19.1 & 0.0 & 110 & 18.6 & 0.0 & 84 & \\
\hline$w^{0}$ & 17.1 & -2.3 & 157 & 22.8 & -3.7 & 169 & 21.2 & -2.6 & 140 & $109^{7}$ \\
\hline \multicolumn{11}{|c|}{$\mathrm{e}^{\mathrm{M}}$ (2-fluoroethanol) } \\
\hline$e^{M} w$ & 22.3 & 0.0 & 166 & 29.5 & 0.0 & 162 & 25.6 & 0.0 & 126 & 105 \\
\hline $\mathrm{we}^{\mathrm{M}}$ & 17.1 & 5.2 & 142 & 24.0 & 5.5 & 143 & 21.7 & 3.9 & 122 & 97 \\
\hline \multicolumn{11}{|c|}{$\mathrm{e}^{\mathrm{D}}$ (2,2-difluoroethanol) } \\
\hline$e^{D} w$ & 22.9 & 0.0 & 176 & 30.0 & 0.0 & 169 & 27.0 & 0.0 & 135 & 115 \\
\hline$w e^{D}$ & 15.9 & 7.0 & 112 & 24.1 & 5.9 & 111 & 21.1 & 5.9 & 92 & \\
\hline \multicolumn{11}{|c|}{$\mathrm{e}^{\mathrm{T}}(2,2,2$-trifluoroethanol $)$} \\
\hline$e^{T} w$ & 24.0 & 0.0 & 193 & 30.6 & 0.0 & 188 & 28.2 & 0.0 & 150 & 128 \\
\hline$w e^{T}$ & 12.0 & 12.0 & 83 & 18.2 & 12.4 & 67 & 17.0 & 11.2 & 52 & \\
\hline
\end{tabular}

different structures are predicted to be much more pronounced for the hetero- than for the homodimers, ${ }^{26-28}$ a fact which can be experimentally observed in the mixed expansion spectra being of comparable complexity under jet-cooled conditions as the pure ones.

On a side note, trial calculations on structures with a donor water molecule attached to the fluorinated methyl group without any further alcohol-water interactions indicate a decreasing stability with proceeding fluorination, which leads us to formulate an increasingly hydrophobic character of the fluoromethyl moieties.

Counterpoise calculations for all structures demonstrate quite drastic BSSE corrections in the MP2 case, on the order of $10 \mathrm{~kJ} \mathrm{~mol}^{-1}$ for the $\mathrm{e}^{\mathrm{z}} \mathrm{w}$ and $6-7 \mathrm{~kJ} \mathrm{~mol}^{-1}$ for the $\mathrm{we}^{\mathrm{z}}$ structures. The magnitude of these corrections shows the limited quality of the chosen Pople basis set, partly due to its size (189 basis functions for the w/ $/ \mathrm{e}^{\mathrm{T}}$ system, compared to 437 for aug-cc-pVTZ). The uncorrected energies, however, prove to perform quite well when compared to CBS limit extrapolations including $\operatorname{CCSD}(\mathrm{T})$ corrections for $\mathrm{e}^{\mathrm{M}} \mathrm{W}$ and $\mathrm{we}^{\mathrm{M}}$ : the electronic MP2/6-311++G(d,p) energy gap matches that of the extrapolation within the accuracy of the calculations $\left(4.0 \mathrm{~kJ} \mathrm{~mol}^{-1}\right.$ for $\mathrm{MP} 2 / 6-311++\mathrm{G}(\mathrm{d}, \mathrm{p})$ vs. $4.3 \mathrm{~kJ} \mathrm{~mol}^{-1}$ at the extrapolated CBS limit). Further information on these extrapolations is available in the ESI. $\uparrow$ It appears that the chosen level of MP2 treatment represents a lucky case of error compensation wherein effects from basis set incompleteness (including BSSE), slightly different geometries and methodical errors ameliorate each other. We thus assume the relative energies yielded at the MP2/6-311++G(d,p) level to be realistic, although the absolute dissociation energies are $3 \mathrm{~kJ} \mathrm{~mol}^{-1}$ too high. Absolute dissociation energies for the B3LYP approach are even closer to the high-level extrapolations (see ESI $\dagger$ ). After adding the D3 dispersion correction, this coincidental agreement is evidently lost. The overshooting by 
6-8 $\mathrm{kJ} \mathrm{mol}^{-1}$ means that these calculations should also be restricted to relative energy discussions.

Table 2 assembles the relative stabilities of the heterodimer structures, $\Delta E_{0}$, and the predicted spectroscopic shifts $-\Delta \omega$ of the donor relative to its free form (i.e., the gauche alcohol for each $\mathrm{e}^{\mathrm{z}} \mathrm{w}$ structure, and the symmetric stretch of water for $\mathrm{we}^{\mathrm{z}}$ ). The calculations confirm the experimental assignments of the dominant mixed dimers in Fig. 1-3 and encourage us to tentatively assign the secondary $3560 \mathrm{~cm}^{-1}$ feature in the $\mathrm{e}^{\mathrm{M}}$ spectra (Fig. 1) to the less stable $\mathrm{we}^{\mathrm{M}}$ structure. The calculated harmonic hydrogen bond shifts overestimate the observed anharmonic ones in a fairly systematic way by 54(3)\% (B3LYP), 49(3)\% (B3LYP-D3) and 18(1)\% (MP2). They all confirm a gradual increase of the red shift with increasing fluorination for $\mathrm{e}^{\mathrm{z}} \mathrm{w}$ and a decreasing shift for $\mathrm{we}^{\mathrm{z}}$ from $z=0$ to $z=\mathrm{M}$. The MP2 results for $\mathrm{e}^{\mathrm{T}}$ compare well with MP2/aug-cc-pVDZ data by Senent et al., ${ }^{5}$ which predict a we ${ }^{\mathrm{T}}-\mathrm{e}^{\mathrm{T}} \mathrm{W}$ energy difference of $9.9 \mathrm{~kJ} \mathrm{~mol}^{-1}$ and analogous red shifts of the donor-OH stretching wavenumbers of $163 \mathrm{~cm}^{-1}$ for $\mathrm{e}^{\mathrm{T}} \mathrm{W}$ and $69 \mathrm{~cm}^{-1}$ for $\mathrm{we}^{\mathrm{T}}$.

Table 3 lists several structural parameters in the $\mathrm{e}^{\mathrm{z}}$ monomers and $\mathrm{e}^{\mathrm{z}} \mathrm{w}$ dimers that are sensitive to hydrogen bond formation. It becomes clear that proceeding fluorination strengthens the primary $\mathrm{OH}$. . O bond, slightly shortening it and forcing it back towards linearity. At the same time, the elongation of the internal $\mathrm{OH}$ bond in the donor fluoroalcohol becomes more pronounced with increasing fluorination. This "straightening" of the primary bond pulls the water molecule away from the fluorine atom, thereby successively (and significantly) elongating the secondary $\mathrm{OH} \cdots \mathrm{F}$ contact. As shown by the trial calculations for exclusive water coordination of the methyl group, this is facilitated by a substantial decrease of the water attraction with increasing fluorination. For $\mathrm{e}^{\mathrm{T}}$, no corresponding local minimum for water coordination was found, in contrast to the $\mathrm{e}^{\mathrm{M}}$ case.

\subsection{Analysis of the fluorination trend}

While one could simply accept the finding that ethanol fluorination consistently switches the donor-acceptor preference for all employed theoretical levels as well as in experiment, this observation deserves deeper analysis in view of its future rational use in related systems. What fraction of the effect may be attributed to the electronic through-bond effect of the fluorine substitution (Table 3)? How can one rationalize the quick saturation of the $\mathrm{e}^{\mathrm{z}} \mathrm{w}$ dissociation energy with increasing fluorination (Table 2)? Can the observed and calculated $\mathrm{OH}$

Table 3 Structural parameters of intermolecular contacts for $e^{z} w$ structures, calculated at the MP2/6-311++G(d,p) level: changes in intramolecular O-H bond length $\left(\Delta r_{\mathrm{OH}}\right)$ and $\mathrm{OH}$ torsional angle $\left(\Delta \tau_{\mathrm{OH}}\right.$, gauche $\left.\approx 60^{\circ}\right)$ relative to the monomers; length of the intermolecular $\mathrm{OH} \ldots \mathrm{O}$ hydrogen bond $\left(r_{\mathrm{OH}} \ldots \mathrm{O}\right)$, the $\mathrm{OH} \ldots \mathrm{O}$ bond angle $\left(\alpha_{\mathrm{OH} \ldots \mathrm{O}}\right)$ and $\mathrm{OH} \ldots \mathrm{F}$ distances $\left(r_{\mathrm{OH} \ldots \mathrm{F}}\right)$

\begin{tabular}{llllll}
\hline & $\Delta r_{\mathrm{OH}} / \mathrm{pm}$ & $\Delta \tau_{\mathrm{OH}} / \mathrm{deg}$ & $r_{\mathrm{OH} \cdots \mathrm{O}} / \mathrm{pm}$ & $\alpha_{\mathrm{OH} \cdots \mathrm{O}} / \mathrm{deg}$ & $r_{\mathrm{OH} \cdots \mathrm{F}} / \mathrm{pm}$ \\
\hline $\mathrm{e}^{0} \mathrm{~W}$ & 0.4 & 0 & 195 & 179 & - \\
$\mathrm{e}^{\mathrm{M}} \mathrm{W}$ & 0.6 & 21 & 191 & 163 & 207 \\
$\mathrm{e}^{\mathrm{D}} \mathrm{W}$ & 0.7 & 17 & 188 & 164 & 227 \\
$\mathrm{e}^{\mathrm{T}} \mathrm{W}$ & 0.8 & 12 & 187 & 169 & 245
\end{tabular}

stretching frequency shifts (Table 2) be taken as an indicator for the primary hydrogen bond strength or for a combined effect of primary and secondary hydrogen bonds? How much of the $\mathrm{OH}$ stretching frequency shift has a structural origin ( $\mathrm{OH}$ bond lengthening in Table 3) and to what extent is it a direct electronic effect of $\mathrm{OH}$ bond softening due to the interaction?

The first analysis concentrates on pure electronic interaction energies without zero-point or relaxation effects, i.e. the energy of the optimized complex is compared to the energy of its two fragments in the absence of their respective binding partner. Throughout this section, we indicate the use of such nonoptimized monomer reference structures taken from the complex by "nopt", as compared to locally optimized ("opt") monomer reference structures. For the dimer structures, full optimization was carried out in all cases. Fig. 6 compares $D_{\mathrm{e}}^{\text {nopt }}$ MP2 dissociation energies as a function of fluorination for the $\mathrm{e}^{\mathrm{z}} \mathrm{w}$ complexes in their most stable gauche orientation and in a $C_{\mathrm{s}}$ symmetry constrained trans orientation of the alcohol, with the fluorinated methyl group oriented such that it conserves the symmetry. While these trans structures are not very stable for fluorinated ethanols, ${ }^{28,31}$ only the energy gain upon water complexation is of interest in this analysis. The trend is quite striking: for ethanol itself, the difference between gauche and trans dissociation energies is negligible, whereas it jumps to $8 \mathrm{~kJ} \mathrm{~mol}^{-1}$ for single fluorination. This is twice as much as the through-bond fluorination effect of $4 \mathrm{~kJ} \mathrm{~mol}^{-1}$ revealed by the trans structures and must be attributed to the cooperative action of the weak $\mathrm{OH} \cdots \mathrm{F}$ contact on the primary $\mathrm{OH} \cdots \mathrm{O}$ bond. The through-bond effect increases steadily with additional fluorination, as revealed by the trans curve in Fig. 6 and as intuitively expected. In contrast, the gauche interaction energy stays constant. Any gain in through-bond polarization of the primary hydrogen bond is fully compensated by a loss in cooperative enhancement by the secondary hydrogen bond. In trifluoroethanol, 3/4 of the interaction energy gain relative to ethanol are due to through-bond polarization and only $1 / 4$ due to the weak secondary contact. Although a more detailed analysis would have to include the electrostatic field changes

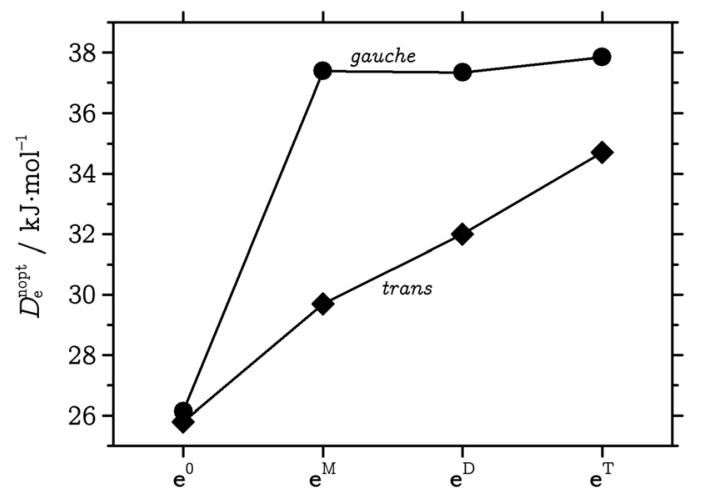

Fig. 6 Electronic dissociation energies $D_{\mathrm{e}}^{\text {nopt }}$ of trans (diamonds) and gauche (circles) $e^{z} w$ structures, showing the steady through-bond effect and the outstanding cooperative enhancement in $\mathrm{e}^{\mathrm{M}}$. 


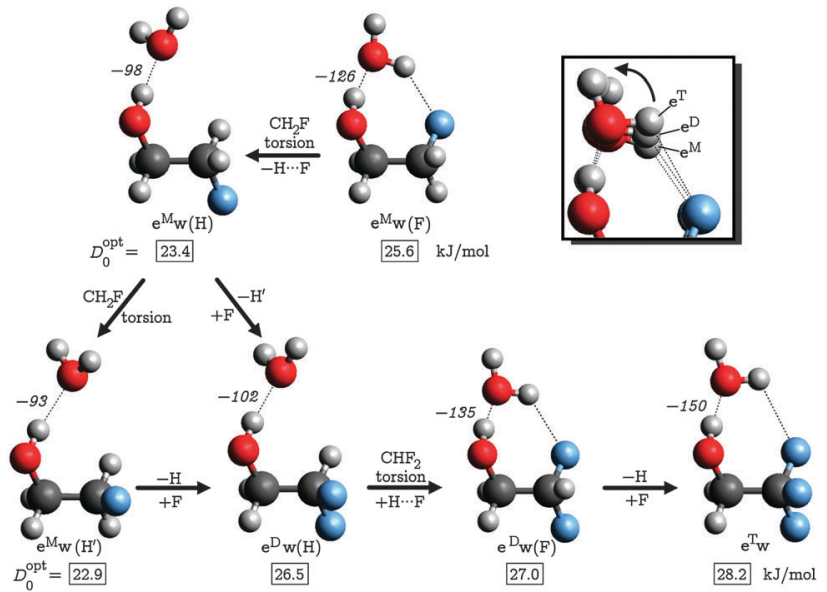

Fig. 7 Stepwise reconstruction of the $\mathrm{OH}$...F contact contribution. Annotated are the predicted red shifts of the fluoroethanol $\mathrm{OH}$ stretching vibration $-\Delta \omega^{\mathrm{opt}}$ (in $\mathrm{cm}^{-1}$, in italics) and the zero-point corrected dissociation energy $D_{0}^{\text {opt }}$ (in $\mathrm{kJ} \mathrm{mol}^{-1}$ ), each relative to the respective fluoroethanol conformer. The inset shows a superposition of the most stable $e^{z} w$ clusters to visualize the "straightening" effect of fluorination on the primary hydrogen bond to water. All calculations at the MP2/6-311++G(d,p) level.

induced by the progressive fluorination, the qualitative finding is robust: $\mathrm{e}^{\mathrm{M}}$ and $\mathrm{e}^{\mathrm{T}}$ bind to water as hydrogen bond donors for very different reasons $-\mathrm{e}^{\mathrm{M}}$ mostly because it offers a cooperative hydrogen bond situation to the water molecule and $\mathrm{e}^{\mathrm{T}}$ largely because its $\mathrm{OH}$ group is a much stronger donor than ethanol. For $\mathrm{e}^{\mathrm{D}}$, the contributions are more balanced.

With this background, it is easy to rationalize the fluorinationconformation pathway shown in Fig. 7 for the MP2 results, quantified in Table 4 for all three computational levels and elaborated in Table 5 as a function of relaxation and zero-pointenergy inclusion. When rotating the fluoromethyl groups, the atom pointing towards the water $(\mathrm{H}$ or $\mathrm{F})$ is added in parentheses to the $\mathrm{e}^{\mathrm{z}} \mathrm{w}$ code. Twisting away the secondary $\mathrm{OH} \cdots \mathrm{F}$ contact in $\mathrm{e}^{\mathrm{M}} \mathrm{w}(\mathrm{F})$ to $\mathrm{e}^{\mathrm{M}} \mathrm{w}(\mathrm{H})$ costs $6.2 \mathrm{~kJ} \mathrm{~mol}^{-1}$ of interaction energy, which are attenuated to $3.1 \mathrm{~kJ} \mathrm{~mol}^{-1}$ by allowing the monomers to relax to their local minimum (Table 5); the structural effect is mostly due to the torsional

Table 4 Dissociation energies $D_{0}^{\mathrm{opt}}$ (in $\mathrm{kJ} \mathrm{mol}^{-1}$ ) and red shifts of $\mathrm{OH}$ stretching vibrations $-\Delta \omega^{\mathrm{opt}}$ (in $\mathrm{cm}^{-1}$ ) along the pathway in Fig. 7. See the text for details on the labeling scheme and associated structures

\begin{tabular}{|c|c|c|c|c|c|c|}
\hline & \multicolumn{2}{|l|}{ B3LYP } & \multicolumn{2}{|c|}{ B3LYP-D3 } & \multicolumn{2}{|l|}{ MP2 } \\
\hline & $D_{0}^{\mathrm{opt}}$ & $-\Delta \omega^{\mathrm{opt}}$ & $D_{0}^{\mathrm{opt}}$ & $-\Delta \omega^{\mathrm{opt}}$ & $D_{0}^{\mathrm{opt}}$ & $-\Delta \omega^{\mathrm{opt}}$ \\
\hline $\mathrm{e}^{\mathrm{M}} \mathrm{w}(\mathrm{F})$ & 22.3 & 166 & 29.5 & 162 & 25.6 & 126 \\
\hline$\downarrow_{-\mathrm{H} \cdots \mathrm{F}}^{\mathrm{CH}_{2} \mathrm{~F} \text { torsion }}$ & $\downarrow-2.6$ & $-34 \downarrow$ & $\downarrow-4.8$ & $-43 \downarrow$ & $\downarrow-2.2$ & $-28 \downarrow$ \\
\hline $\mathrm{e}^{\mathrm{M}} \mathrm{w}(\mathrm{H})$ & 19.7 & 132 & 24.7 & 119 & 23.4 & 98 \\
\hline$\downarrow \mathrm{CH}_{2} \mathrm{~F}$ torsion & $\downarrow-0.6$ & $-11 \downarrow$ & $\downarrow-0.9$ & $-4 \downarrow$ & $\downarrow-0.5$ & $-5 \downarrow$ \\
\hline $\mathrm{e}^{\mathrm{M}} \mathrm{w}\left(\mathrm{H}^{\prime}\right)$ & 19.1 & 121 & 23.8 & 115 & 22.9 & 93 \\
\hline$\downarrow+\mathrm{F}$ & $\downarrow+3.7$ & $+26 \downarrow$ & $\downarrow+4.0$ & $+8 \downarrow$ & $\downarrow+3.6$ & $+9 \downarrow$ \\
\hline $\mathrm{e}^{\mathrm{D}} \mathrm{w}(\mathrm{H})$ & 22.8 & 147 & 27.8 & 123 & 26.5 & 102 \\
\hline $\begin{array}{l}\downarrow_{+\mathrm{H} \cdots \mathrm{F}}^{\mathrm{CHF}_{2} \text { torsion }} \\
+\mathrm{H}\end{array}$ & $\downarrow+0.1$ & $+29 \downarrow$ & $\downarrow+2.2$ & $+46 \downarrow$ & $\downarrow+0.5$ & $+33 \downarrow$ \\
\hline $\mathrm{e}^{\mathrm{D}} \mathrm{w}(\mathrm{F})$ & 22.9 & 176 & 30.0 & 169 & 27.0 & 135 \\
\hline$\downarrow+\mathrm{F}$ & $\downarrow+1.1$ & $+17 \downarrow$ & $\downarrow+0.6$ & $+19 \downarrow$ & $\downarrow+1.2$ & $+15 \downarrow$ \\
\hline$e^{T} w$ & 24.0 & 193 & 30.6 & 188 & 28.2 & 150 \\
\hline
\end{tabular}

Table 5 MP2/6-311++G(d,p) electronic dissociation energies $D_{\mathrm{e}}$ (in $\mathrm{kJ} \mathrm{mol}^{-1}$ ) and harmonic red shifts in $\mathrm{OH}$ stretching wavenumbers $-\Delta \omega$ (in $\mathrm{cm}^{-1}$ ) along the pathway in Fig. 7. $\Delta E^{\text {struc }}=D_{\mathrm{e}}^{\text {nopt }}-D_{\mathrm{e}}^{\mathrm{opt}}$ is the overall relaxation energy from the dimer to the monomer optimum of the alcohol and water fragments. See the text for details on the labeling scheme and associated structures

\begin{tabular}{llllll}
\hline & $D_{\mathrm{e}}^{\text {opt }}$ & $-\Delta \omega^{\text {opt }}$ & $D_{\mathrm{e}}^{\text {nopt }}$ & $-\Delta \omega^{\text {nopt }}$ & $\Delta E^{\text {struc }}$ \\
\hline $\mathrm{e}^{\mathrm{M}} \mathrm{W}(\mathrm{F})$ & 34.0 & 126 & 37.4 & 46 & 3.4 \\
$\downarrow^{\mathrm{CH}} \mathrm{F}$ torsion & $\downarrow-3.1$ & $-28 \downarrow$ & $\downarrow-6.2$ & $-18 \downarrow$ & \\
$\mathrm{e}^{\mathrm{M}} \mathrm{W}(\mathrm{H})$ & 30.9 & 98 & 31.2 & 28 & 0.3 \\
$\downarrow \mathrm{CH}_{2} \mathrm{~F}$ torsion & $\downarrow-0.2$ & $-5 \downarrow$ & $\downarrow-0.2$ & $-1 \downarrow$ & \\
$\mathrm{e}^{\mathrm{M}} \mathrm{W}\left(\mathrm{H}^{\prime}\right)$ & 30.7 & 93 & 31.0 & 27 & 0.3 \\
$\downarrow-\mathrm{H}$ & $\downarrow+3.9$ & $+9 \downarrow$ & $\downarrow+4.1$ & $+2 \downarrow$ & \\
$\mathrm{e}^{\mathrm{D}} \mathrm{W}(\mathrm{H})$ & 34.6 & 102 & 35.1 & 29 & 0.5 \\
$\downarrow \downarrow_{+}^{\mathrm{CHF}}$ torsion & $\downarrow+0.5$ & $+33 \downarrow$ & $\downarrow+2.2$ & $+18 \downarrow$ & \\
$\mathrm{e}^{\mathrm{D}} \mathrm{W}(\mathrm{F})$ & 35.1 & 135 & 37.3 & 47 & 2.2 \\
$\downarrow-\mathrm{H}$ & $\downarrow+1.3$ & $+15 \downarrow$ & $\downarrow+0.5$ & $+2 \downarrow$ & \\
$\mathrm{e}^{\mathrm{T}} \mathrm{F}$ & 36.4 & 150 & 37.8 & 49 & 1.4 \\
\end{tabular}

displacement of the $\mathrm{OH}$ group in the dimer (see Table 3). Addition of zero-point energy, which favors floppy structures, further dampens the effect to $2.2 \mathrm{~kJ} \mathrm{~mol}^{-1}$ ( $c f$. Table 4). Further torsion to $\mathrm{e}^{\mathrm{M}} \mathrm{w}\left(\mathrm{H}^{\prime}\right)$ has little effect, as expected if electrostatic field action through space is less important. The effect of replacing one $\mathrm{H}$ by $\mathrm{F}$ increases the binding of the water molecule by $4.1 \mathrm{~kJ} \mathrm{~mol}^{-1}$, quite independent of whether relaxation and zero-point energy are included or not. This is the predicted through-bond effect. When the secondary interaction is switched on in $\mathrm{e}^{\mathrm{D}}$ by fluoromethyl group torsion, the interaction energy only increases by $2.2 \mathrm{~kJ} \mathrm{~mol}^{-1}$ (Table 5), further attenuated below $1 \mathrm{~kJ} \mathrm{~mol}^{-1}$ by monomer relaxation and zero-point motion. The net $\mathrm{e}^{\mathrm{M}} \mathrm{w}(\mathrm{F}) \rightarrow \mathrm{e}^{\mathrm{D}} \mathrm{w}(\mathrm{F})$ effect of the second fluorine is therefore seen to be very modest. It is virtually zero on the interaction energy level and below $2 \mathrm{~kJ} \mathrm{~mol}^{-1}$ including structural and zero-point energies. Introduction of the third fluorine also shows effects on the sub-2 $\mathrm{kJ} \mathrm{mol}^{-1}$ level, in continuation of the fluorination trend. This reflects the geometric trend (Table 3) in the upper-right inset of Fig. 7 of a receding secondary interaction along with the increase of the primary hydrogen bond strength. Table 4 shows that the discussed effects are not specific to the MP2 calculations. In slightly modulated form, the through-bond enhancement of the $\mathrm{OH}$...O interaction is always nearly compensated by the net effect of breaking the secondary contact in $\mathrm{e}^{\mathrm{M}}$ and reforming it in $\mathrm{e}^{\mathrm{D}}$.

Returning to Table 2, one can see that the inversion of the donor-acceptor preference of water (indicated by positive values of $\Delta E_{0}$ ) as a function of fluorination results from the double action of through-bond acidification and cooperativity in $\mathrm{e}^{\mathrm{M}}$, which tends to saturate in $\mathrm{e}^{\mathrm{D}}$ for the above-mentioned reasons and is again boosted for $\mathrm{e}^{\mathrm{T}}$ because the $\mathrm{CH} \cdots \mathrm{O}$ hydrogen bond option in the competing $\mathrm{we}^{\mathrm{z}}$ structure is lost.

Turning now to the trends in $\mathrm{OH}$ stretching wavenumber shifts, we dissect the overall shift observed upon heterodimer formation into two contributions. One is due to the distortion of the $\mathrm{OH}$ oscillator by the interaction. First, a torsion of the $\mathrm{OH}$ group out of its minimum position (see Table 3) increases the harmonic $\mathrm{OH}$ stretching frequency, as demonstrated for $\mathrm{e}^{0} .^{15}$ This effect is overcompensated by a positive shift in the 
$\mathrm{OH}$ bond length, which leads to a lower curvature (due to anharmonicity) and thus also harmonic wavenumber than at the minimum position. The second, electronic contribution is due to a softening of the oscillator by the interaction with the water molecule. For a linear perturbation by this interaction, the effect on the wavenumber vanishes; ${ }^{32}$ only if the perturbation is non-linear, a further wavenumber shift arises. There are two more or less equivalent ways to decompose the harmonic shift into these contributions for a localized oscillator such as in fluorinated ethanol. One is to evaluate the harmonic force field of the monomer in the dimer structure, ignoring the gradient. The other involves a local 1D analysis of the free and the distorted monomer in terms of the curvature at the respective structures obtained by $\pm 0.001 \AA$ elongation, after scaling the reduced mass to reproduce the harmonic results of the optimized monomers. We have tested both approaches and find them to yield equivalent structural shifts within $10 \mathrm{~cm}^{-1}$ for all relevant structures, similar to earlier calculations on $\mathrm{e}^{0}$ homodimers. $^{33}$ This agreement indicates that the $\mathrm{OH}$ oscillator is sufficiently local for a 1D description in these systems. A detailed description of our approach is available in the ESI. $†$ In the following, only the results from the $1 \mathrm{D}$ analysis are given. Table 6 summarizes the results of the dissection of the total harmonic red shift $-\Delta \omega^{\text {opt }}$ into the purely electronic part $-\Delta \omega^{\text {nopt }}$ and the structural part (difference of the two numbers).

For the series of $C_{\mathrm{s}}$-symmetric trans structures with water as the hydrogen bond acceptor, the total harmonic MP2 red shift of the dimers relative to the opt monomers increases with fluorination from $91 \mathrm{~cm}^{-1}$ in $\mathrm{e}^{0}$ (where it is not the most stable complex) over $118 \mathrm{~cm}^{-1}$ in $\mathrm{e}^{\mathrm{M}}$ to $156 \mathrm{~cm}^{-1}$ in $\mathrm{e}^{\mathrm{T}}$. The electronic effect increases more sharply, tripling on an absolute scale from $20 \mathrm{~cm}^{-1}$ to $61 \mathrm{~cm}^{-1}$. This is clear evidence for the intrinsically increasing strength of the hydrogen bond between the alcohol and the accepting water in the absence of a cooperative $\mathrm{OH} \cdots \mathrm{F}$ contact. In the more stable gauche alcohol donor complexes shown on the right-hand side of Table 6 , the MP2 shift increases from $84 \mathrm{~cm}^{-1}$ for $\mathrm{e}^{0}$ over $126 \mathrm{~cm}^{-1}$ for $\mathrm{e}^{\mathrm{M}}$ to $150 \mathrm{~cm}^{-1}$ for $\mathrm{e}^{\mathrm{T}}$ (see Table 2). Now, the electronic contribution is almost constant for all three fluorination stages. This reflects the compensating trends of increasing primary hydrogen bond strength and decreasing cooperativity with the secondary contact along the fluorination sequence, when purely electronic effects on the stiffness of the mode are analyzed. In the total

Table 6 Electronic dissociation energies $D_{\mathrm{e}}^{\text {nopt }}$ (in $\mathrm{kJ} \mathrm{mol}^{-1}$ ) and harmonic red shifts of $\mathrm{OH}$ stretching wavenumbers $-\Delta \omega$ (in $\mathrm{cm}^{-1}$ ) of trans and gauche $\mathrm{e}^{\mathrm{z}} \mathrm{W}$ complexes. "opt" and "nopt" indicate the reference monomer structures such that $-\Delta \omega^{\text {nopt }}$ represents the electronic (non-structural) contribution to the total red shift $-\Delta \omega^{\text {opt }}$

\begin{tabular}{|c|c|c|c|c|c|c|}
\hline & \multicolumn{3}{|l|}{ trans } & \multicolumn{3}{|c|}{ gauche } \\
\hline & $D_{\mathrm{e}}^{\text {nopt }}$ & $-\Delta \omega^{\mathrm{opt}}$ & $-\Delta \omega^{\text {nopt }}$ & $D_{\mathrm{e}}^{\text {nopt }}$ & $-\Delta \omega^{\mathrm{opt}}$ & $-\Delta \omega^{\text {nopt }}$ \\
\hline$e^{0} w$ & 25.8 & 91 & 20 & 26.1 & 84 & 33 \\
\hline$e^{M_{w}}$ & 29.7 & 118 & 31 & 37.4 & 126 & 46 \\
\hline$e^{D_{w}}$ & 32.0 & 129 & 45 & 37.3 & 135 & 47 \\
\hline$e^{T} w$ & 34.7 & 156 & 61 & 37.8 & 150 & 49 \\
\hline
\end{tabular}

shifts listed in Tables 4 and 5 , this effect is diluted by the overlaid structural shift, but still noticeable.

At this point, we are again in the position to better understand the fluorination-conformation pathway shown in Fig. 7 on the spectroscopic scale. The predicted MP2 red shift of $126 \mathrm{~cm}^{-1}$ in $\mathrm{e}^{\mathrm{M}} \mathrm{W}$ decreases by $22 \%$ when removing the secondary contact, but the electronic shift contribution actually drops by $40 \%$ (Table 5) reflecting the loss of cooperativity. Further torsion into the $\mathrm{H}^{\prime}$ position and the second fluorine have little effect $\left( \pm 5 \mathrm{~cm}^{-1}\right)$ and some of that is actually structural in character, as Table 5 shows. The electronic contribution $-\Delta \omega^{\text {nopt }}$ changes only by $1-2 \mathrm{~cm}^{-1}$. Reestablishment of a secondary contact in $\mathrm{e}^{\mathrm{D}_{\mathrm{W}}}$ raises the shift by $30 \%$ (60\% for the electronic contribution), bringing it back to where it was in $\mathrm{e}^{\mathrm{M}} \mathrm{w}(\mathrm{F})$ despite a reduced cooperativity. While the overall energetics are quite sensitive to the primary hydrogen bond, the electronic component of the red shift weighs the combined action of the primary hydrogen bond and cooperativity more heavily. However, one should not dismiss its sensitivity to non-bonded $\mathrm{O} \cdots \mathrm{F}$ contacts and other stereoelectronic effects.

To summarize, the primary hydrogen bond becomes increasingly stronger with fluorination, as indicated by the interaction energies $D_{\mathrm{e}}^{\text {nopt }}$ and electronic red shifts $-\Delta \omega^{\text {nopt }}$ of the trans structures (see Fig. 6 and Table 6). Conversely, the interaction energies and red shifts of the gauche dimers show no distinct trend for the three fluoroalcohols (Fig. 6 and Table 5). The reason is a decreasing importance of the cooperative secondary contact along the fluorination sequence, which counteracts the increasing strength of the primary hydrogen bond. The experimentally observable trends in the red shifts only emerge from structural relaxation of the monomers. In view of such subtle effects, it is reassuring that Table 4 demonstrates a fair regularity of the behavior along the pathway among the three different computational approaches employed in this work.

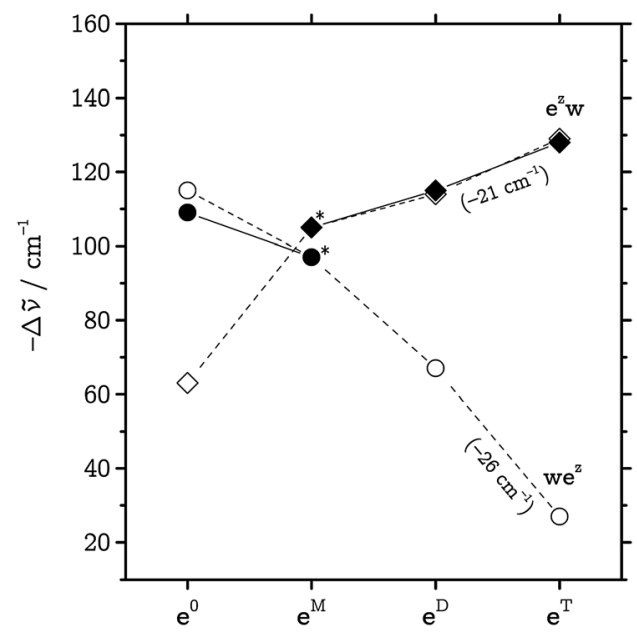

Fig. 8 Red shifts $-\Delta \tilde{\nu}$ of the donor $\mathrm{OH}$ bands as a function of fluorination for $w^{z}$ (circles) and $e^{z} w$ (diamonds) structures. Solid symbols represent experimental values, hollow symbols MP2/6-311++G(d,p) calculations. The calculated data were shifted by the values given in parentheses to match the experimental ones marked with asterisks. 


\subsection{Calculated and observed dimer shifts}

Independent of the dissection of the fluorination trends presented in the preceding section, Fig. 8 summarizes calculated and observed donor red shifts for the four experimentally characterized $\mathrm{e}^{\mathrm{z}} \mathrm{w}$ and $\mathrm{we}^{\mathrm{z}}$ clusters. For better comparability, the calculated MP2 values were superimposed on the observed ones by a uniform shift for each series, using the assigned $\mathrm{e}^{\mathrm{M}}$ bands as "anchors". It is then obvious that, albeit being affected by basis set incompleteness and neglect of anharmonicity, the calculations perform well in predicting the spectroscopic behavior of the alcohol-donor structures, including our tentative assignment of the $\mathrm{we}^{\mathrm{M}}$ band. The fact that the metastable $\mathrm{e}^{0} \mathrm{w}$ dimer remained unobserved ${ }^{7}$ despite a smaller predicted energy gap (2.6 vs. $3.9 \mathrm{~kJ} \mathrm{~mol}^{-1}$ at the MP2/6-311++G(d,p) level) indicates lower isomerization barriers in the nonfluorinated case.

\section{Conclusions and outlook}

The vibrational spectra of jet-cooled mono-, di- and trifluoroethanol $\left(\mathrm{e}^{\mathrm{z}}, \mathrm{z}=\mathrm{M}, \mathrm{D}, \mathrm{T}\right)$ in mixtures with water $(\mathrm{w})$ were obtained in the $\mathrm{O}-\mathrm{H}$ stretching region. Assignments of dominant spectral features to mixed $\mathrm{e}^{\mathrm{z}} \mathrm{w}$ dimers with water as the hydrogen bond acceptor were made. This is in contrast to the ethanol-water case ${ }^{9}$ where water acts as the donor. The corresponding metastable $\mathrm{we}^{\mathrm{z}}$ isomer was tentatively assigned for $\mathrm{e}^{\mathrm{M}}$ only. Although ${ }^{18} \mathrm{O}$ isotope substitution ${ }^{34}$ could be invoked to secure the assignment, the overall agreement with moderately shifted simple quantum-chemical calculations (Fig. 8) already provides compelling evidence, as does the predicted energetic sequence of the donor-acceptor isomers.

While the switch in donor-acceptor preference of water with the introduction of fluorine atoms into the ethanol molecule is thus experimentally established, the rationalization of this switch is somewhat less straightforward. It is found to be the consequence of a sudden, but quickly saturating stabilization of the water acceptor structure with progressive fluorination, followed by a particularly unstable donor role towards trifluoroethanol due to the lack of polarized terminal $\mathrm{CH}$ bonds. The saturation results from a subtle balance between increasing donor strength and decreasing cooperative enhancement by an $\mathrm{OH} \cdots \mathrm{F}$ contact. Thus, the pharmaceutically important trifluoromethyl group ${ }^{35,36}$ is revealed as strongly polarizing but weakly engaging in intermolecular interactions with water. This persists at the level of bulk liquid mixtures. ${ }^{37}$ Partitioning of the $\mathrm{OH}$ stretching shift into a structural component from monomer distortion and a smaller but very sensitive electronic component from bond softening proves to be helpful in emphasizing the spectral trends.

Although higher levels of electronic structure treatment and anharmonic calculations are always desirable, the analysis presented in this study provides robust evidence for the switch in hydrogen bond pattern by ethanol fluorination and for its dominant reason. On the other hand, our results contribute to a better understanding of the peptide structure-modulation effects in trifluoroethanol-water mixtures obtained from molecular dynamics simulations. ${ }^{38}$

\section{Acknowledgements}

We thank the DFG (German Research Foundation) for financial support (SFB 357 and Su121/4). The experimental fluoroethanol data are part of the PhD thesis of T. Scharge. ${ }^{29}$ The ethanolwater spectra were provided by M. Nedić. ${ }^{7}$

\section{References}

1 H.-J. Schneider, Angew. Chem., Int. Ed., 2009, 48, 3924-3977.

2 B. E. Smart, J. Fluorine Chem., 2001, 109, 3-11.

3 K. Müller, C. Faeh and F. Diederich, Science, 2007, 317, 1881-1886.

4 H.-J. Schneider, Chem. Sci., 2012, 3, 1381-1394.

5 M. L. Senent, A. Nino, C. Munoz-Caro, Y. G. Smeyers, R. Dominguez-Gomez and J. M. Orza, J. Phys. Chem. A, 2002, 106, 10673-10680.

6 A. Berkessel and J. A. Adrio, J. Am. Chem. Soc., 2006, 128, 13412-13420.

7 M. Nedić, T. N. Wassermann, R. W. Larsen and M. A. Suhm, Phys. Chem. Chem. Phys., 2011, 13, 14050-14063.

8 C. Emmeluth, V. Dyczmons and M. A. Suhm, J. Phys. Chem. A, 2006, 110, 2906-2915.

9 M. Nedić, T. N. Wassermann, Z. Xue, P. Zielke and M. A. Suhm, Phys. Chem. Chem. Phys., 2008, 10, 5953-5956.

10 E. E. Fileti, P. Chaudhuri and S. Canuto, Chem. Phys. Lett., 2004, 400, 494-499.

11 E. E. Fileti, M. A. Castro and S. Canuto, Chem. Phys. Lett., 2008, 452, 54-58.

12 T. S. van Erp and E. J. Meijer, J. Chem. Phys., 2003, 118, 8831-8840.

13 B. Oliveira and M. Vasconcellos, THEOCHEM, 2006, 774, 83-88.

14 M. Mons, E. G. Robertson, L. C. Snoek and J. P. Simons, Chem. Phys. Lett., 1999, 310, 423-432.

15 T. N. Wassermann and M. A. Suhm, J. Phys. Chem. A, 2010, 114, 8223-8233.

16 A. Giardini, F. Rondino, G. Cattenacci, A. Paladini, S. Piccirillo, M. Satta and M. Speranza, Chem. Phys. Lett., 2007, 435, 230-235.

17 A. Burakowski, J. Glinski, B. Czarnik-Matusewicz, P. Kwoka, A. Baranowski, K. Jerie, H. Pfeiffer and N. Chatziathanasiou, J. Phys. Chem. B, 2012, 116, 705-710.

18 N. Borho, M. A. Suhm, K. Le Barbu-Debus and A. Zehnacker, Phys. Chem. Chem. Phys., 2006, 8, 4449-4460.

19 M. A. Suhm and F. Kollipost, Phys. Chem. Chem. Phys., 2013, 15, 10702-10721.

20 F. Neese, Wiley Interdiscip. Rev.: Comput. Mol. Sci., 2012, 2, 73-78.

21 M. J. Frisch, G. W. Trucks, H. B. Schlegel, G. E. Scuseria, M. A. Robb, J. R. Cheeseman, G. Scalmani, V. Barone, B. Mennucci, G. A. Petersson, H. Nakatsuji, M. Caricato, X. Li, H. P. Hratchian, A. F. Izmaylov, J. Bloino, G. Zheng, 
J. L. Sonnenberg, M. Hada, M. Ehara, K. Toyota, R. Fukuda, J. Hasegawa, M. Ishida, T. Nakajima, Y. Honda, O. Kitao, H. Nakai, T. Vreven, J. A. Montgomery, Jr., J. E. Peralta, F. Ogliaro, M. Bearpark, J. J. Heyd, E. Brothers, K. N. Kudin, V. N. Staroverov, R. Kobayashi, J. Normand, K. Raghavachari, A. Rendell, J. C. Burant, S. S. Iyengar, J. Tomasi, M. Cossi, N. Rega, J. M. Millam, M. Klene, J. E. Knox, J. B. Cross, V. Bakken, C. Adamo, J. Jaramillo, R. Gomperts, R. E. Stratmann, O. Yazyev, A. J. Austin, R. Cammi, C. Pomelli, J. W. Ochterski, R. L. Martin, K. Morokuma, V. G. Zakrzewski, G. A. Voth, P. Salvador, J. J. Dannenberg, S. Dapprich, A. D. Daniels, Ö. Farkas, J. B. Foresman, J. V. Ortiz, J. Cioslowski and D. J. Fox, Gaussian 09, Revision C.01, Gaussian Inc., Wallingford, CT, 2009.

22 S. Grimme, J. Comput. Chem., 2004, 25, 1463-1473.

23 S. Grimme, J. Comput. Chem., 2006, 27, 1787-1799.

24 S. Grimme, J. Antony, S. Ehrlich and H. Krieg, J. Chem. Phys., 2010, 132, 154104.

25 I. D. Mackie and G. A. DiLabio, J. Chem. Phys., 2011, 135, 134318.

26 T. Scharge, C. Emmeluth, T. Häber and M. A. Suhm, J. Mol. Struct., 2006, 786, 86-95.
27 T. Scharge, T. N. Wassermann and M. A. Suhm, Z. Phys. Chem., 2008, 222, 1407-1452.

28 T. Scharge, C. Cézard, P. Zielke, A. Schütz, C. Emmeluth and M. A. Suhm, Phys. Chem. Chem. Phys., 2007, 9, 4472-4490.

29 T. Scharge, Schwache Bindungen mit starker Wirkung: Konformation und Aggregation von halogenierten Alkoholen, Sierke Verlag, Göttingen, 2008.

30 J. Flaud and C. Camy-Peyret, J. Mol. Spectrosc., 1974, 51, 142-150.

31 M. B. de Amorim, Int. J. Quantum Chem., 2008, 108, 2550-2556.

32 S. Y. Liu and C. E. Dykstra, J. Phys. Chem., 1986, 90, 3097-3103.

33 C. Emmeluth, V. Dyczmons, T. Kinzel, P. Botschwina, M. A. Suhm and M. Yáñez, Phys. Chem. Chem. Phys., 2005, 7, 991-997.

34 J. Zischang, J. J. Lee and M. A. Suhm, J. Chem. Phys., 2011, 135, 061102.

35 M. Zanda, New J. Chem., 2004, 28, 1401-1411.

36 W. K. Hagmann, J. Med. Chem., 2008, 51, 4359-4369.

37 A. Cooney and K. Morcom, J. Chem. Thermodyn., 1988, 20, 735-741.

38 D. Roccatano, G. Colombo, M. Fioroni and A. E. Mark, Proc. Natl. Acad. Sci. U. S. A., 2002, 99, 12179-12184. 